\title{
Two-dimensional conformal field theories with matrix-valued level
}

\author{
Ali Nassar \\ Department of Physics, University of Science and Technology, Zewail City of Science \\ and Technology, 12588 Giza, Egypt
}

\begin{abstract}
We introduce a new class of two dimensional conformal field theories by extending Wess-Zumino-Witten (WZW) models to chiral algebras with matrixvalued levels. The new CFTs are based on holomorphic currents with an operator product expansion characterized by a positive integer-valued matrix $K_{A B}$. We use the Sugawara construction to compute the energy-momentum tensor, the central charge, and the spectrum of conformal dimensions of the CFTs based on this algebra. We also construct a set of genus-1 characters and show that they fulfil a representation of the modular group $\operatorname{SL}(2, \mathbb{Z})$ up to a modular anomaly.
\end{abstract}

\section{Introduction}

The study of 2D conformal field theories (CFTs) is motivated by their importance in string theory and critical phenomena (see [1, 3] ). They describe the world-sheet dynamics of perturbative string theory and correspond to string backgrounds which solves the classical equations of motion [9, 10]. In many statistical mechanical systems, CFTs describe renormalization group fixed points when the system exhibits scale invariance and long-range correlations. One of the major unsolved problems in this field is the construction and classification of all 2D CFTs (see [6, 11] for a review and references therein). An important structure in 2D CFT is the chiral algebra which gives rise to infinite-dimensional chiral symmetries. This chiral algebra could be the Virasoro algebra or one of its extensions. The Virasoro algebra $\operatorname{Vir} \oplus \overline{\operatorname{Vir}}$ is the

Email address: anassar@zewailcity.edu.eg (Ali Nassar) 
chiral algebra which underlies any 2D CFT and is generated by the energymomentum tensor $T(z), \bar{T}(\bar{z})$ which is a spin-2 field. The chiral algebra can get extended by the inclusion of integer- or half-integer-spin holomorphic fields [1 [2]. The possible extension of the Virasoro algebra have been classified by Zamolodchikov [13]. In this paper, we consider the extension of the Virasoro algebra by spin-1 holomorphic currents $J_{A}^{a}(z)$ with an operator product expansion (OPE) characterized by a matrix-valued level $K_{A B}$. The zero modes of these currents generate the horizontal subalgebra $g^{\oplus_{N}}=g \oplus \cdots \oplus g$ where $g$ is a compact simple Lie algebra. We will denote this algebra by $\hat{g}_{N, K}$. For a diagonal matrix-level $k_{A} \delta_{A B}$ the algebra $\hat{g}_{N, K}$ reduces to a WessZumino-Witten (WZW) model based on $g \oplus \cdots \oplus g$. The algebra $\hat{g}_{N, K}$ is not the central extension of the loop algebra of $g^{\oplus_{N}}$ due to a cohomological obstruction. We will use the Sugawara construction to construct an energymomentum tensor as a bilinear in the currents $J_{A}^{a}(z)$. This leads to 2D CFTs with new values for the central and charge conformal dimensions. A set of specialized genus-1 characters will be constructed and we will show that they transform under the modular group $\mathrm{SL}(2, \mathbb{Z})$ up to a modular anomaly proportional to the above mentioned cohomological obstruction.

This paper is organized as follows: In Section (2), we introduce the matrix-valued algebra $\hat{g}_{N, K}$ and compute its central charge and conformal dimensions. In Section (3), we write down the genus-1 characters and show that they transform under the modular group $\operatorname{SL}(2, \mathbb{Z})$ up to a modular anomaly. In Section (44), we apply our results to the algebra $\widehat{s u(2)}{ }_{N, K}$.

\section{The matrix-level algebra $\hat{g}_{N, K}$}

One source of chiral algebras is the affine Kac-Moody current algebras based on the central extension of some Lie algebra [7], e.g., the level $k$ WZW models based on $\hat{g}_{k}[12]$. For a compact simply-connected Lie group $G$ with a simple Lie algebra $g$, the Kac-Moody currents $J^{a}(z)$ of $\hat{g}_{k}$ have an operator product expansion (OPE)

$$
J^{a}(z) J^{b}(w) \sim \frac{i f_{a b c} J^{c}(w)}{z-w}+\frac{k \delta^{a b}}{(z-w)^{2}} .
$$

\footnotetext{
${ }^{1}$ Half-integer spin fields generate superconformal symmetries.
} 
This OPE gives rise to following modes algebra

$$
\left[J_{m}^{a}, J_{n}^{b}\right]=i f_{a b c} J_{m+n}^{c}+k \Omega\left(J_{m}^{a}, J_{n}^{b}\right), \quad \Omega\left(J_{m}^{a}, J_{n}^{b}\right)=m \delta^{a b} \delta_{m+n, 0},
$$

where $k \in \mathbb{Z}_{+}$.

The central extension term $\Omega\left(J_{m}^{a}, J_{n}^{b}\right)$ defines a 2-cocycle on the loop group of $g$, i.e., it satisfies

$$
\begin{gathered}
\Omega\left(J_{m}^{a}, J_{n}^{b}\right)=-\Omega\left(J_{n}^{b}, J_{m}^{a}\right) \\
\Omega\left(\left[J_{m}^{a},\left[J_{n}^{b}, J_{r}^{c}\right]\right]\right)+\Omega\left(\left[J_{r}^{c},\left[J_{m}^{a}, J_{n}^{b}\right]\right]\right)+\Omega\left(\left[J_{n}^{b},\left[J_{r}^{c}, J_{m}^{a}\right]\right]\right)=0 .
\end{gathered}
$$

The energy-momentum tensor is constructed by the Sugawara construction

$$
T(z)=\gamma J^{a}(z) J^{a}(z),
$$

where $\gamma$ is fixed by the requirement that $J^{a}(z)$ has conformal dimension equal to one. This leads to

$$
\gamma=\frac{1}{2(k+h)}
$$

where $h$ is the dual Coxeter number of $g$. Once $\gamma$ is fixed, one can use the TT OPE to compute the central charge of the theory

$$
T(z) T(w) \sim \frac{c / 2}{(z-w)^{4}}+\frac{2 T(w)}{(z-w)^{2}}+\frac{\partial T(w)}{(z-w)}
$$

which gives

$$
c(k)=\frac{k \operatorname{dim} g}{k+h} .
$$

The Sugawara construction can be extended to the case of semisimple Lie algebras which are direct sums $g^{\oplus_{N}}=g_{1} \oplus \cdots \oplus g_{N}$ of simple algebras $g_{A}$. The total energy-momentum tensor will be the sum of the Sugawara energy-momentum tensors for each simple factor. The fact that the individual energy-momentum tensors commute means that the total central charge will be the sum of the individual central charges

$$
C=\sum_{A=1}^{N} c\left(k_{A}\right)=\sum_{A=1}^{N} \frac{k_{A} \operatorname{dim} g_{A}}{k_{A}+h_{A}},
$$

where $k_{A} \in \mathbb{Z}_{+}$, $\operatorname{dim} g_{A}$, and $h_{A}$ are the level, the dimension, and the dual Coxeter number of $\hat{g}_{k_{A}}^{A}$, respectively. 
We propose a matrix-level Kac-Moody algebra based on $g^{\oplus_{N}}$ which we denote by $\hat{g}_{N, K}$. We take $K_{A B}$ to be the intersection form of a positive integral2 lattice $\Gamma_{K}$, i.e., $K_{A B} \in \mathbb{Z}_{+}$. The inverse $K^{A B}$ is the intersection form of the dual lattice $\Gamma_{K}^{*}$.

We will denote the currents by $J_{A}^{a}$, where $a$ is the Lie algebra index $a=1, \cdots, \operatorname{dim}(g)$ and $A=1, \cdots, N=\operatorname{rank}(K)$. The Lie algebra of $g^{\oplus N}$ takes the form

$$
\left[J_{A}^{a}, J_{B}^{b}\right]=i f_{a b c} J_{B}^{c} \delta_{A B} .
$$

We assume the following OPE of the currents

$$
J_{A}^{a}(z) J_{B}^{b}(w) \sim \frac{i f_{a b c} J_{B}^{c}(w) \delta_{A B}}{z-w}+\frac{\delta^{a b} K_{A B}}{(z-w)^{2}} .
$$

An important consistency check which we will use in our subsequent calculations is the concept of factorization, i.e., when $K_{A B}=k_{A} \delta_{A B}$ (diagonal matrix level) all our formulas should boil down to the familiar algebra

$$
\hat{g}_{N, \vec{k}}=\hat{g}_{k_{1}} \oplus \cdots \oplus \hat{g}_{k_{N}} .
$$

We use the Sugawara construction to find the energy-momentum tensor

$$
T(z)=\sum_{A, B=1}^{N} G^{A B}\left(J_{A}^{a} J_{B}^{a}\right)(z),
$$

where the summation convention is used on the Lie algebra index $a$. Here $G^{A B}$ is such that the currents $J_{M}^{a}(z)$ have conformal dimension one

$$
T(w) J_{M}^{a}(z) \sim \frac{J_{M}^{a}(z)}{(w-z)^{2}}+\frac{\partial J_{M}^{a}(z)}{(w-z)} .
$$

\footnotetext{
${ }^{2}$ See the discussion after
} 
To compute this OPE we use

$T(w) J_{M}^{a}(z)$

$$
\begin{aligned}
= & \sum_{A, B=1}^{N} G^{A B}\left(J_{A}^{b} J_{B}^{b}\right)(w) J_{M}^{a}(z) \\
= & \frac{1}{2 \pi i} \oint_{w} \frac{d x}{x-w} \sum_{A, B=1}^{N}\left[G^{A B} J_{M}^{a} \overparen{(z) J_{A}^{b}}(x) J_{B}^{b}(w)+G^{A B} J_{A}^{b}(x) J_{M}^{a}(z) J_{B}^{b}(w)\right] \\
= & \frac{1}{2 \pi i} \oint_{w} \frac{d x}{x-w} \sum_{A, B=1}^{N}\left\{G^{A B}\left[\frac{i f_{a b c} \delta_{M A} J_{A}^{c}(x)}{(z-x)}+\frac{K_{M A} \delta_{a b}}{(z-x)^{2}}\right] J_{B}^{b}(w)\right. \\
& \left.+G^{A B} J_{A}^{b}(x)\left[\frac{i f_{a b c} \delta_{M B} J_{B}^{c}(w)}{(z-w)}+\frac{K_{M B} \delta_{a b}}{(z-w)^{2}}\right]\right\} \\
= & \frac{1}{2 \pi i} \oint_{w} \frac{d x}{x-w} \sum_{A, B=1}^{N}\left\{\frac{-f_{a b c} f_{c b d} G^{A B} \delta_{M A} \delta_{A B} J_{B}^{d}(w)}{(z-x)(x-w)}+\frac{i f_{a b c} G^{A B} \delta_{M A}\left(J_{A}^{c} J_{B}^{b}\right)(w)}{(z-x)}\right. \\
& \left.+\frac{G^{A B} K_{M A} J_{B}^{a}(w)}{(z-x)^{2}}+\frac{G^{A B} K_{M B} J_{A}^{a}(x)}{(z-w)^{2}}+\frac{i f_{a b c} G^{A B} \delta_{M B}\left(J_{A}^{b} J_{B}^{c}\right)(w)}{(z-w)}\right\}
\end{aligned}
$$

Using

$$
f_{a b c} f_{c b d}=-2 h \delta_{a d}, \quad \sum_{B} G^{M B} f_{a b c}\left[\left(J_{M}^{c} J_{B}^{b}\right)+\left(J_{M}^{b} J_{B}^{c}\right)\right]=0
$$

Then

$$
\begin{aligned}
\sum_{A, B=1}^{N} & G^{A B}\left(J_{A}^{b} J_{B}^{b}\right)(w) J_{M}^{a}(z) \\
& =\frac{1}{2 \pi i} \oint_{w} \frac{d x}{x-w} \sum_{A, B=1}^{N}\left[\frac{G^{A B} K_{M A} J_{B}^{a}(x)}{(z-x)^{2}}+\frac{2 h G^{M M} J_{M}^{a}(w)}{(z-x)(x-w)}+\frac{G^{A B} K_{M B} J_{A}^{a}(w)}{(z-w)^{2}}\right] \\
& =\sum_{A, B=1}^{N} \frac{G^{A B} K_{M A} J_{B}^{a}(w)+2 h G^{M M} J_{M}^{a}(w)+G^{A B} K_{M B} J_{A}^{a}(w)}{(z-w)^{2}}
\end{aligned}
$$


We look for a $G^{A B}$ of the form

$$
G^{A B}=\gamma^{A} K^{A B}
$$

where $K^{A B}=K_{A B}^{-1}$ and $\gamma^{A}$ will be chosen in such a way to force $J_{M}^{a}$ to have conformal dimension equal to one. Using this form of $G^{A B}$ in (16) we get

$$
\begin{aligned}
\gamma^{A} & =\frac{1}{2\left(1+h K^{A A}\right)} \\
G^{A B} & =\frac{K^{A B}}{2\left(1+h K^{A A}\right)} .
\end{aligned}
$$

The energy-momentum tensor now reads

$$
T(z)=\sum_{A, B=1}^{N} \frac{K^{A B}}{2\left(1+h K^{A A}\right)}\left(J_{A}^{a} J_{B}^{a}\right)(z) .
$$

The $T J$ OPE now reads

$$
T(z) J_{A}^{a}(w) \sim \frac{J_{A}^{a}(w)}{(z-w)^{2}}+\frac{\partial J_{A}^{a}(w)}{(z-w)} .
$$

The OPE of $T(z)$ with itself can be similarly computed

$$
\begin{aligned}
T(z) T(w) & \\
= & \sum_{A, B=1}^{N} G^{A B} \frac{1}{2 \pi i} \oint_{w} \frac{d x}{x-w}\left[T\left(\overrightarrow{(z) J_{A}^{a}}(x) J_{B}^{a}(w)+J_{A}^{a}(x) T(z) J_{B}^{a}(w)\right]\right. \\
= & \sum_{A, B=1}^{N} G^{A B} \frac{1}{2 \pi i} \oint_{w} \frac{d x}{x-w}\left\{\left[\frac{J_{A}^{a}(x) J_{B}^{a}(w)}{(z-x)^{2}}+\frac{\partial J_{A}^{a}(x) J_{B}^{a}(w)}{(z-x)}\right]\right. \\
& \left.+\left[\frac{J_{A}^{a}(x) J_{B}^{a}(w)}{(z-w)^{2}}+\frac{J_{A}^{a}(x) \partial J_{B}^{a}(w)}{(z-w)}\right]\right\} \\
= & \sum_{A, B=1}^{N} G^{A B} \frac{1}{2 \pi i} \oint_{w} \frac{d x}{x-w}\left\{\left[\frac{\operatorname{dim} g K_{A B}}{(z-x)^{2}(x-w)^{2}}+\frac{\left(J_{A}^{a} J_{B}^{a}\right)(w)}{(z-x)^{2}}\right.\right. \\
& \left.\left.-\frac{2 \operatorname{dim} g K_{A B}}{(z-x)(x-w)^{3}}+\frac{\left(\partial J_{A}^{a} J_{B}^{a}\right)(w)}{(z-x)}\right]+\left[\frac{\left(J_{A}^{a} J_{B}^{a}\right)(w)}{(z-w)^{2}}+\frac{\left(J_{A}^{a} \partial J_{B}^{a}\right)(w)}{(z-w)}\right]\right\},
\end{aligned}
$$


where we used

$$
\delta^{a a}=\operatorname{dim} g, \quad J_{A}^{a}(x) \partial J_{B}^{a}(w) \sim \frac{2 \operatorname{dim} g K_{A B}}{(x-w)^{3}}+\left(J_{A}^{a} \partial J_{B}^{a}\right)(w) .
$$

We also used the identity

$$
\frac{1}{2 \pi i} \oint \frac{d x}{(x-w)^{n}} \frac{F(w)}{(z-x)^{m}}=\frac{(n+m-2) !}{(n-1) !(m-1) !} \frac{F(w)}{(z-w)^{n+m-1}} .
$$

Using

$$
\sum_{A, B=1}^{N} G^{A B} K_{A B}=\sum_{A, B=1}^{N} \frac{K^{A B} K_{A B}}{2\left(1+h K^{A A}\right)}=\sum_{A}^{N} \frac{1}{2\left(1+h K^{A A}\right)} .
$$

Then (22) can be written as

$$
T(z) T(w) \sim \frac{C(K) / 2}{(z-w)^{4}}+\frac{2 T(w)}{(z-w)^{2}}+\frac{\partial T(w)}{(z-w)}
$$

where the central charge $C(K)$ is

$$
C(K)=\sum_{A=1}^{N} \frac{\operatorname{dim} g}{1+h K^{A A}}
$$

For $N=1$ and $K=k^{-1}$, we recover the usual value of $C$

$$
C(k)=\frac{k \operatorname{dim} g}{h+k}
$$

For the diagonal case $K_{A B}=k_{A} \delta_{A B}$, the central charge factorizes as expected

$$
C(K)=\sum_{A=1}^{N} c\left(k_{A}\right)=\sum_{A=1}^{N} \frac{k_{A} \operatorname{dim} g}{h+k_{A}} .
$$

The Abelian limit of $\hat{g}_{N, K}$ corresponds to the algebra $\hat{u}_{N, K}=\hat{u}(1)_{K}^{\oplus_{N}}$, where $K$ is an $N \times N$ matrix-valued level. This algebra was studied in [5] and its modular invariant partition functions were given. In this case, the operator product expansion of the currents takes the form

$$
J_{A}(z) J_{B}(w) \sim \frac{K_{A B}}{(z-w)^{2}} .
$$


This algebra was related to strings on complex multiplication tori in [8]. In [4], Gannon studied the classification of the modular invariant partition functions of the algebra $\widehat{s u(2)} k_{1} \oplus \cdots \oplus \widehat{s u(2)} k_{N}$ which in our notation corresponds to the algebra $\widehat{s u(2)}{ }_{N, K}$ with a diagonal $K$ matrix $K_{A B}=k_{A} \delta_{A B}$.

Now we go back to the algebra (10) and study it in more details. By expanding the currents $J_{A}^{a}(z)$ in modes

$$
J_{A}^{a}(z)=\sum_{m \in \mathbb{Z}} \frac{J_{A}^{a}(m)}{z^{m+1}} .
$$

The requirement $J_{A}^{a}(z)^{\dagger}=J_{A}^{a}(z)$ leads to $J_{A}^{a}(m)^{\dagger}=J_{A}^{a}(-m)$. We can write the OPE (10) in terms of the modes

$$
\left[J_{A}^{a}(m), J_{B}^{b}(n)\right]=i f_{a b c} J_{B}^{c}(m+n) \delta_{A B}+m K_{A B} \delta_{a b} \delta_{m+n, 0} .
$$

The central extension term $\Omega\left(J_{A}^{a}(m), J_{B}^{b}(n)\right)=m K_{A B} \delta_{a b} \delta_{m+n, 0}$ satisfies

$$
\begin{gathered}
\Omega\left(J_{A}^{a}(m), J_{B}^{b}(n)\right)=-\Omega\left(J_{B}^{b}(n), J_{A}^{a}(m)\right) \\
\Omega\left(\left[J_{A}^{a}(m),\left[J_{B}^{b}(n), J_{C}^{c}(r)\right]\right]\right)+\Omega\left(\left[J_{C}^{c}(r),\left[J_{A}^{a}(m), J_{B}^{b}(n)\right]\right]\right) \\
+\Omega\left(\left[J_{B}^{b}(n),\left[J_{C}^{c}(r), J_{A}^{a}(m)\right]\right]\right) \\
=i f_{a b c} \delta_{m+n+r, 0}\left[m \delta_{B C} K_{A C}+n \delta_{A B} K_{C B}+r \delta_{C A} K_{A B}\right]
\end{gathered}
$$

This is equal zero if $K$ is a diagonal matrix and is non-zero for generic $K$. Hence, there is a cohomological obstruction for $\Omega\left(J_{A}^{a}(m), J_{B}^{b}(n)\right)$ to define a 2-cocycle on the loop group of $g^{\oplus N}$ and hence the algebra $\hat{g}_{N, K}$ is not the affine extension of the loop algebra of $g^{\oplus N}$. However, as pointed out in [5], the important structure in CFT is the chiral algebra itself. The chiral algebra (10) is well defined and gives rise to unitary CFTs with a set of genus-1 characters with definite modular transformation properties and a modular covariant partition function. The failure of the 2-cocycle condition will not affect the OPE of the primary fields (defined by (36)) and as such will not interfere with the fundamental requirement of associativity of their correlation functions.

The modes of the energy-momentum tensor are given by

$$
\mathbb{L}_{n}=\sum_{A, B=1}^{N} \sum_{m \in \mathbb{Z}} \frac{K^{A B}}{2\left(1+h K^{A A}\right)}: J_{A}^{a}(m) J_{B}^{a}(n-m):
$$


These modes satisfy the Virasoro algebra

$$
\left[\mathbb{L}_{n}, \mathbb{L}_{m}\right]=(n-m) \mathbb{L}_{n+m}+\frac{C}{12}\left(n^{3}-n\right) \delta_{n+m, 0}
$$

where the central charge $C$ is given by (27).

Primary fields are characterized by the following OPE with the currents

$$
\begin{aligned}
J_{A}^{a}(z) \Phi_{\Lambda, \Xi}(w, \bar{w}) & \sim \frac{-t_{\lambda_{A}}^{a} \Phi_{\Lambda, \Xi}(w, \bar{w})}{z-w} \\
\bar{J}_{A}^{a}(\bar{z}) \Phi_{\Lambda, \Xi}(w, \bar{w}) & \sim \frac{\Phi_{\Lambda, \Xi}(w, \bar{w}) t_{\xi_{A}}^{a}}{z-w}
\end{aligned}
$$

This defines a primary field in the representation $(\Lambda, \Xi)$ of the horizontal subalgebra $\oplus_{A=1}^{N} g_{A}$ where

$$
(\Lambda ; \Xi)=\left(\oplus_{A} \lambda_{A} ; \oplus_{A} \xi_{A}\right)
$$

A primary field $\Phi_{\Lambda, \Xi}$ will give rise to a primary state $\left|\Phi_{\Lambda, \Xi}\right\rangle$ and the action of the current modes on primary states is

$$
\begin{aligned}
J_{A}^{a}(0)\left|\Phi_{\Lambda, \Xi}\right\rangle & =-t_{\lambda_{A}}^{a}\left|\Phi_{\Lambda, \Xi}\right\rangle, \\
J_{A}^{a}(n)\left|\Phi_{\Lambda, \Xi}\right\rangle & =0, \quad n>0 .
\end{aligned}
$$

These states are also Virasoro primary states

$$
\begin{aligned}
& \mathbb{L}_{0}\left|\Phi_{\Lambda, \Xi}\right\rangle=h_{\Lambda}\left|\Phi_{\Lambda, \Xi}\right\rangle, \\
& \mathbb{L}_{n}\left|\Phi_{\Lambda, \Xi}\right\rangle=0, \quad n>0 .
\end{aligned}
$$

where $h_{\Lambda}$ is the holomorphic conformal dimension of the primary field $\Phi_{\Lambda, \Xi}(w, \bar{w})$ and can be computed by noting that the action of $\mathbb{L}_{0}$ on primary states takes the simple form

$$
\mathbb{L}_{0}=\sum_{A, B=1}^{N} \frac{K^{A B}}{2\left(1+h K^{A A}\right)}: J_{A}^{a}(0) J_{B}^{a}(0):
$$


This leads to

$$
\begin{aligned}
\mathbb{L}_{0}\left|\Phi_{\Lambda, \Xi}\right\rangle & =\sum_{A, B=1}^{N} \frac{K^{A B}}{2\left(1+h K^{A A}\right)} t_{\lambda_{A}}^{a} t_{\lambda_{B}}^{a}\left|\Phi_{\Lambda, \Xi}\right\rangle \\
& =\left(\sum_{A=1}^{N} \frac{K^{A A}}{2\left(1+h K^{A A}\right)} t_{\lambda_{A}}^{a} t_{\lambda_{A}}^{a}+\sum_{\substack{A, B=1 \\
A \neq B}}^{N} \frac{K^{A B}}{2\left(1+h K^{A A}\right)} t_{\lambda_{A}}^{a} t_{\lambda_{B}}^{a}\right)\left|\Phi_{\Lambda, \Xi}\right\rangle \\
& =\left(\sum_{A=1}^{N} \frac{K^{A A}}{2\left(1+h K^{A A}\right)} C\left(\lambda_{A}\right)+\sum_{\substack{A, B=1 \\
A \neq B}}^{N} \frac{K^{A B}}{2\left(1+h K^{A A}\right)} H_{\lambda_{A}}^{i} H_{\lambda_{B}}^{i}\right)\left|\Phi_{\Lambda, \Xi}\right\rangle \\
& =\left(\sum_{A=1}^{N} \frac{K^{A A}}{2\left(1+h K^{A A}\right)} C\left(\lambda_{A}\right)+\sum_{\substack{A, B=1 \\
A \neq B}}^{N} \frac{K^{A B}}{2\left(1+h K^{A A}\right)} \lambda_{A} \cdot \lambda_{B}\right)\left|\Phi_{\Lambda, \Xi}\right\rangle
\end{aligned}
$$

where $C\left(\lambda_{A}\right)$ is the quadratic Casimir eigenvalue in the representation $\lambda_{A}$ and $H_{\lambda_{A}}^{i}$ are the elements of the Cartan subalgebra of $g_{A}$ in the representation $\lambda_{A}$ and we defined

$$
\lambda_{A} \cdot \lambda_{B}=\lambda_{A}^{i} \lambda_{B}^{i}
$$

Hence the holomorphic conformal dimension of the field $\Phi_{\Lambda, \Xi}(w, \bar{w})$ is given by

$$
h_{\Lambda}=\sum_{A=1}^{N} \frac{K^{A A}}{2\left(1+h K^{A A}\right)} C\left(\lambda_{A}\right)+\sum_{\substack{A, B=1 \\ A \neq B}}^{N} \frac{K^{A B}}{2\left(1+h K^{A A}\right)} \lambda_{A} \cdot \lambda_{B} .
$$

Now we use the requirement of unitarity to derive some constraints on the matrix-level $K_{A B}$ and on the set of allowed representations. By restricting the algebra (32) to a subset of the generators one gets

$$
\left[J_{A}^{a}(m), J_{A}^{a}(-m)\right]=m K_{A A} .
$$

Using the fact that $J_{A}^{a}(m)^{\dagger}=J_{A}^{a}(-m)$ to define the state

$$
\left|\Psi_{A}^{a}(m)\right\rangle=J_{A}^{b}(-m)|0\rangle, \quad m>0,
$$

where

$$
J_{A}^{b}(m)|0\rangle=0, \quad m>0 .
$$


Then (44) can be written as

$$
\left\langle\Psi_{A}^{a}(m) \mid \Psi_{A}^{a}(m)\right\rangle=m K_{A A} .
$$

Hence, unitarity forces us to chose

$$
K_{A A}>0 .
$$

By using the Cartan-Weyl basis for the algebra (32) and in particular

$$
\left[E_{A}^{\alpha}(1), E_{A}^{-\alpha}(-1)\right]=\alpha \cdot H_{A}(0)+K_{A A}
$$

One can derive the usual WZW bound

$$
\lambda_{A} \cdot \theta \leq K_{A A},
$$

where $\theta$ is the longest root of $g$.

Hence the positivity and integrality conditions on the diagonal elements $K_{A A}$ are required for the unitarity of the corresponding CFTs and in order to have a finite number of highest weight states as explained by (48) and (50), respectively. It would be interesting to study extensions of these models for general $K_{A B}$ subject only to the ondition $K_{A A} \in \mathbb{Z}_{+}$.

Also, in a unitary CFT, the central charge and the conformal dimensions can't take negative values. This will lead to further constraints on $K^{A B}$ and can be analyzed in different cases.

\section{Genus-1 characters}

The total Hilber space $\mathcal{H}$ of our CFT will split into subspaces $\mathcal{H}_{\Lambda} \otimes \mathcal{H}_{\Xi}$

$$
\mathcal{H}=M_{\Lambda \Xi} \mathcal{H}_{\Lambda} \otimes \mathcal{H}_{\Xi},
$$

where $M_{\Lambda \Xi} \in \mathbb{Z}_{+}$counts the multiplicity of the $\mathcal{H}_{\Lambda} \otimes \mathcal{H}_{\Xi}$ in the CFT spectrum. On top of each one of the subspaces $\mathcal{H}_{\Lambda} \otimes \mathcal{H}_{\Xi}$ sets a primary state $|\Lambda, \bar{\Xi}\rangle$. The other members of $\mathcal{H}_{\Lambda}$ will be generated by acting on $|\Lambda\rangle$ with the lowering operators of $\hat{g}_{N, K}$. The level- $M$ descendent state is given by 3

$$
|\Lambda, M\rangle=\prod_{i} J_{A_{i}}^{a_{i}}\left(-m_{i}\right)|\Lambda\rangle, \quad \sum_{i} m_{i}=M .
$$

\footnotetext{
${ }^{3}$ Here we only consider the holomorphic part of the CFT.
} 
Using the fact that

$$
\left[\mathbb{L}_{0}, J_{A}^{a}(m)\right]=-m J_{A}^{a}(m)
$$

one can compute the conformal dimensions of $|\Lambda, M\rangle$

$$
\mathbb{L}_{0}|\Lambda, M\rangle=\left(h_{\Lambda}+M\right)|\Lambda, M\rangle \text {. }
$$

To simplify the computation of the genus- 1 characters we write

$$
\mathbb{L}_{0}=L_{0}+\tilde{L}_{0}
$$

where

$$
\begin{aligned}
& L_{0}=\sum_{A=1}^{N} \frac{K^{A A}}{2\left(1+h K^{A A}\right)}: J_{A}^{a}(0) J_{A}^{a}(0):, \\
& \tilde{L}_{0}=\sum_{\substack{A, B=1 \\
A \neq B}}^{N} \frac{K^{A B}}{2\left(1+h K^{A A}\right)}: J_{A}^{a}(0) J_{B}^{a}(0): .
\end{aligned}
$$

These operators have the following action on primary states

$$
L_{0}|\Lambda\rangle=h_{\Lambda}^{0}|\Lambda\rangle, \quad \tilde{L}_{0}|\Lambda\rangle=\tilde{h}_{\Lambda}|\Lambda\rangle, \quad h_{\Lambda}=h_{\Lambda}^{0}+\tilde{h}_{\Lambda},
$$

where

$$
h_{\Lambda}^{0}=\sum_{A=1}^{N} \frac{K^{A A}}{2\left(1+h K^{A A}\right)} C\left(\lambda_{A}\right), \quad \tilde{h}_{\Lambda}=\sum_{\substack{A, B=1 \\ A \neq B}}^{N} \frac{K^{A B}}{2\left(1+h K^{A A}\right)} \lambda_{A} \cdot \lambda_{B}
$$

Using the the fact that

$$
\left[L_{0}, \tilde{L}_{0}\right]=0
$$

Hence

$$
\left[\mathbb{L}_{0}, L_{0}\right]=0 .
$$

This equation simply means that $\mathbb{L}_{0}$ and $L_{0}$ can be diagonalized simultaneously although with different eigenvalues. This observation simplifies the construction of the genus-one characters

$$
\tilde{\chi}_{\Lambda}=\operatorname{Tr}_{\mathcal{H}_{\Lambda}} q^{\mathbb{L}_{0}-c / 24}
$$


Using the action of $\mathbb{L}_{0}$ on the states $|\Lambda, M\rangle \in \mathcal{H}_{\Lambda}$ given by (154), the genus-one characters can be witten as

$$
\begin{aligned}
\tilde{\chi}_{\Lambda} & =\sum_{M} q^{h_{\Lambda}+M-c / 24} \\
& =\sum_{M} q^{h_{\Lambda}^{0}+\tilde{h}_{\Lambda}+M-c / 24} \\
& =q^{\tilde{h}_{\Lambda}} \sum_{M} q^{h_{\Lambda}^{0}+M-c / 24} \\
& =q^{\tilde{h}_{\Lambda}} \chi_{\Lambda}
\end{aligned}
$$

where

$$
\chi_{\Lambda}=\sum_{M} q^{h_{\Lambda}^{0}+M-c / 24}=\operatorname{Tr}_{\mathcal{H}_{\Lambda}} q^{L_{0}-c / 24}
$$

is the affine characters of the diagonal matrix-level algebra $\hat{g}_{k_{1}} \oplus \cdots \oplus \hat{g}_{k_{N}}$ and is given by

$$
\chi_{\Lambda}=\chi_{\lambda_{1}}^{k_{1}} \times \cdots \times \chi_{\lambda_{N}}^{k_{N}} .
$$

These characters have the following modular transformations 4

$$
\chi_{\Lambda}(-1 / \tau)=\mathbb{S}_{\Lambda \Sigma} \chi_{\Sigma}(\tau), \quad \chi_{\Lambda}(-1 / \tau)=\mathbb{T}_{\Lambda \Sigma} \chi_{\Sigma}(\tau),
$$

where

$$
\mathbb{S}_{\Lambda \Sigma}=S_{\lambda_{1} \sigma_{1}}^{k_{1}} \otimes \cdots \otimes S_{\lambda_{N} \sigma_{N}}^{k_{N}}, \quad \mathbb{T}_{\Lambda \Sigma}=T_{\lambda_{1} \sigma_{1}}^{k_{1}} \otimes \cdots \otimes T_{\lambda_{N} \sigma_{N}}^{k_{N}},
$$

where $S_{\lambda_{1} \sigma_{1}}^{k_{1}}$ and $T_{\lambda_{1} \sigma_{1}}^{k_{1}}$ are the modular matrices of the algebra $\hat{g}_{k_{1}}$.

Looking back at (61) and since $\tilde{\chi}_{\Lambda}=q^{\tilde{h}_{\Lambda}} \chi_{\Lambda}$, one learns that the matrixlevel characters $\tilde{\chi}_{\Lambda}$ fulfils a representation of the modular group $S L(2, \mathbb{Z})$ up to a modular anomaly given by $\tilde{h}_{\Lambda}$. It is interesting to note that the modular anomaly $\tilde{h}_{\Lambda}$ vanishes for a diagonal matrix level $K$, i.e., when there is no cohomological obstruction for $\hat{g}_{N, K}$ to define an affine extension of the loop algebra of $g^{\oplus N}$.

\footnotetext{
${ }^{4}$ Here we only consider the specialized characters.
} 


\section{The algebra $\widehat{s u(2)}_{N, K}$}

To illustrate the results of the previous sections, we study the simplest kind of matrix-level algebras which is based on $s u(2) \oplus \cdots \oplus s u(2)$. To derive a unitarity bound on the set of allowed representations we define the state

$$
\left|\Theta_{\Lambda}\right\rangle=J_{A}^{-}(-1)|\Lambda\rangle
$$

The norm of this state can be computed using the mode algebra (32)

$$
\left\langle\Theta_{\Lambda} \mid \Theta_{\Lambda}\right\rangle=\left\langle\Lambda\left|J_{A}^{+}(1) J_{A}^{-}(-1)\right| \Lambda\right\rangle=\left(K_{A A}-\lambda_{A}\right) .
$$

Hence the set of allowed representations should satisfy

$$
\lambda_{A} \leq K_{A A}
$$

The spectrum of conformal dimensions of the matrix-level algebra $\widehat{s u(2)} \widehat{N, K}_{1}$ is given by

$$
h_{\Lambda}=\sum_{A=1}^{N} \frac{K^{A A}}{\left(1+h K^{A A}\right)} \frac{\lambda_{A}}{2}\left(\frac{\lambda_{A}}{2}+1\right)+\sum_{\substack{A, B=1 \\ A \neq B}}^{N} \frac{K^{A B}}{2\left(1+h K^{A A}\right)}\left(\frac{\lambda_{A} \lambda_{B}}{4}\right),
$$

where $\lambda_{A}$ is the Dynkin label of the highest weight state. Written in terms of the spin $j_{A}=\lambda_{A} / 2$ of the highest weight state, the above formula can be written as

$$
h_{\Lambda}=\sum_{A=1}^{N} \frac{K^{A A}}{\left(1+h K^{A A}\right)} j_{A}\left(j_{A}+1\right)+\sum_{\substack{A, B=1 \\ A \neq B}}^{N} \frac{K^{A B}}{2\left(1+h K^{A A}\right)}\left(j_{A} j_{B}\right) .
$$

For $\widehat{s u(2)}_{2, K}$ with the matrix level

$$
K=\left(\begin{array}{ll}
1 & 1 \\
1 & 2
\end{array}\right)
$$

The central charge is

$$
C(K)=\frac{8}{5}
$$


Notice that this central charge is not the same as the one for the algebra $\widehat{s u(2)}{ }_{1} \oplus \widehat{s u(2)}{ }_{2}$ (corresponding to a diagonal matrix-level $\operatorname{diag}(1,2)$ ) which is equal to $5 / 2$.

The set of allowed representations of $\widehat{s u(2)}_{2, K}$ is given by

$$
P_{K}=\{(0 ; 0),(0 ; 1),(1 ; 0),(1 ; 1),(0 ; 2),(1 ; 2)\}
$$

The spectrum of holomorphic conformal dimensions is given by

$$
h_{(0 ; 0)}=0, h_{(0 ; 1)}=\frac{1}{4}, h_{(1 ; 0)}=\frac{3}{10}, h_{(1 ; 1)}=\frac{17}{60}, h_{(0 ; 2)}=\frac{2}{3}, h_{(1 ; 2)}=\frac{13}{30} .
$$

Hence, the algebra $\widehat{s u(2)}{ }_{2, K}$ also has different values of the holomorphic conformal dimensions.

\section{Conclusion}

We studied an affine extension of the algebra $g^{\oplus_{N}}=g \oplus \cdots \oplus g$ by spin-1 currents $J_{A}^{a}(z)$ with an OPE characterized by a matrix-valued level $K_{A B}$. The Sugawara construction were used to construct an energy-momentum tensor based on $J_{A}^{a}(z)$. This leads to a new classe of $2 \mathrm{D}$ conformal field theories with new values of the central charge and conformal dimensions. This algebra gives the familiar WZW models as a special case when the matrix-valued level is diagonal. This affine extension is not the central extension of the loop algebra of $g^{\oplus_{N}}$ due to a cohomological obstruction which vanishes for diagonal $K$ matrices. A set of genus-1 characters were constructed which fulfils a representation of the modular group up to a modular anomaly which vanishes for diagonal $K$.

\section{Acknowledgement}

I am grateful to Mark Walton for useful discussions. I thank the annonymus refree for the valuable comments. This research is supported by the CFP at Zewail city of Science and Technology and by the STDF project 13858 .

\section{References}

[1] A. A. Belavin, A. M. Polyakov, and A. B. Zamolodchikov. Infinite conformal symmetry in two-dimensional quantum field theory. Nucl. Phys., B241:333-380, 1984. 
[2] P. Bouwknegt and K. Schoutens. $W$ symmetry in conformal field theory. Phys. Rept., 223:183-276, 1993.

[3] P. Di Francesco, P. Mathieu, and D. Sénéchal. Conformal Field Theory. Springer, 1997.

[4] T. Gannon. Towards a classification of $S U(2) \times \cdots \times S U(2)$ modular invariant partition functions. J. Math. Phys., 36:675-706, 1995.

[5] T. Gannon. $U(1)^{m}$ modular invariants, $N=2$ minimal models, and the quantum Hall effect. Nucl. Phys., B491:659-688, 1997.

[6] T. Gannon. Monstrous moonshine and the classification of CFT. 1999.

[7] V. G. Kac. Infinite Dimensional Lie Algebras. Cambridge University Press, 1990.

[8] A. Nassar and M. A. Walton. Rational conformal field theory and matrix level for strings on a torus. Can. J. Phys., 92:65-70, 2014.

[9] J. Polchinski. String Theory. Vol. 1: An Introduction to the Bosonic String. Cambridge University Press, 1998.

[10] J. Polchinski. String Theory. Vol. 2: Superstring Theory and Beyond. Cambridge University Press, 1998.

[11] K. Wendland. Moduli spaces of unitary conformal field theories. $\mathrm{PhD}$ thesis, Bonn University. Ph.D. Thesis, 2000.

[12] E. Witten. Nonabelian bosonization in two-dimensions. Commun. Math. Phys., 92:455-472, 1984.

[13] A. B. Zamolodchikov. Infinite Additional Symmetries in TwoDimensional Conformal Quantum Field Theory. Theor. Math. Phys., 65:1205-1213, 1985. 